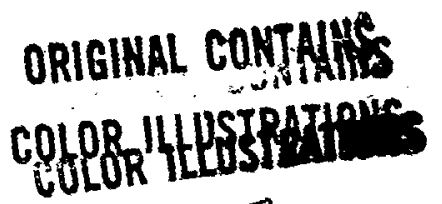

$$
\begin{gathered}
\text { COOUERRED } \\
7715 \\
p-14
\end{gathered}
$$

a reprint from Applied Optics

111231) QUANTITATIVE

N96-19070

RAINBOW SCHLIEREN DEFLECTOMEY) $14 \mathrm{P}$

(NASA. Lewis Research Center 


\title{
Quantitative rainbow schlieren deflectometry
}

\author{
Paul S. Greenberg, Robert B. Klimek, and Donald R. Buchele
}

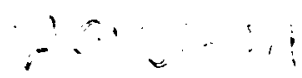

\begin{abstract}
In the rainbow schlieren apparatus, a continuously graded rainbow filter is placed in the back focal plane of the decollimating lens. Refractive-index gradients in the test section thus appear as gradations in hue rather than irradiance. A simple system is described wherein a conventional color CCD array and video digitizer are used to quantify accurately the color attributes of the resulting image, and hence the associated ray deflections. The present system providesi a sensitivity comparable with that of conventional interferometry, while being simpler to implement and less sensitive to mechanical misalignment.

Key words: Schlieren, deflectometry, refractive index
\end{abstract}

\section{Introduction}

Of the many methods for visualizing refractive-index inhomogeneities in transparent media, the schlieren method is one of the oldest and simplest. As an instrument, a schlieren apparatus is sensitive to transverse refractive-index gradients in the test section. These gradients cause incoming light rays to undergo angular deviations, which are in turn encoded by means of selective interaction with a spatial filter (also referred to as the schlieren stop). Depending on the type of spatial filter that is used, these angular deviations may be subsequently visualized as gradations in irradiance, color, fringe (actually grid lines) position, or contrast. Details on the various forms of spatial filters, optical system configurations, and applications have been reported extensively. ${ }^{1-3}$

A generic schlieren system in its simplest form is depicted in Fig. 1. An input mirror or lens is used to collimate the output of the source. The spectral properties and spatial extent of the source are dependent on the specific form of schlieren stop being utilized. After traversing the test section, this collimated light is refocused by the decollimating mirror (or lens) onto the spatial filter and is finally imaged onto a camera or other photographic medium. Notice that two simultaneous real images are formed by the apparatus; an image of the source aperture is

P. S. Greenberg and R. B. Klimek are with the NASA Lewis Research Center, Cleveland, Ohio 44135. D. R. Buchele is with the ADF Corporation, Cleveland, Ohio 44135.

Received 11 July 1994; revised manuscript received 11 October 1994.

0003-6935/95/193810-13\$06.00/0.

- 1995 Optical Society of America. formed on the plane of the spatial filter, which is also the Fourier transform plane of the test section, while an image of the test section is formed by the camera lens onto the photographic medium or imaging array. The optics preceding the camera retransform the output, thus recovering a spatially filtered inverted real image of the test section.

From the physical optics viewpoint, the Fourier shift theorem explains the action of the device: perturbations to the phase of the incident wave front (i.e., ray deflections) caused by refractive-index inhomogeneities cause the image of the source to be displaced laterally in the plane of the spatial filter. The magnitudes and directions of the resulting displacements are thereby revealed by the interaction of the source image with the spatially dependent properties of the filter. Thus the instrument provides, by means of a suitably chosen encoding scheme, a measurement of the quantity

$$
\theta_{\text {exit }} \approx \tan \theta_{\text {exit }}=\int_{L} \nabla n \mathrm{~d} l .
$$

The line integral is evaluated over the region of the test section where the refractive-index gradients are nonvanishing. In general, it is desirable to invert the above relationship to obtain local values for the refractive index from measurements of the deflected angle, $\theta_{\text {exit }}$. The local values of the refractive index are then in turn related to physical quantities of interest such as temperature or density.

The schlieren method has, in general, been employed as a qualitative tool for the visualization of refractive-index perturbations in gases and liquids. Quantitative approaches based on resulting irradiance distributions have been reported but are some- 


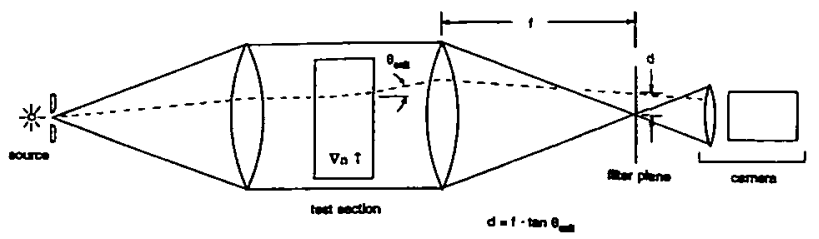

Fig. 1. Schematic drawing of a simple schlieren apparatus.

what difficult to apply in practice. Primarily this is due to the inhomogeneous absorption of light within the test section, focusing of the incident light caused by optical imperfections or object asymmetry (i.e., shadowgraphy), the subtleties in the diffraction of the source image by the spatial filter, and inherent nonlinearities in the recording medium itself. The advent of linear, solid-state imaging arrays represent a significant advance relative to their nonlinear film-based counterparts; however, the other complications remain.

The continuously graded color filter, or rainbow filter, ${ }^{4}$ provides a simple solution to the aforementioned difficulties. The filter possesses no sharp edges or discontinuities; hence diffraction of the source image by the filter is essentially absent. Because refractive-index inhomogeneities are manifest as gradations in color attributes rather than irradiance distributions, numerous problems such as nonuniform absorption or second-order effects (shadowgraphy) are absent as well. The extended source required by conventional knife-edge methods is now relaxed, in effect decoupling the extent of the source image from the reciprocal relationship between sensitivity and dynamic range. ${ }^{5}$ Furthermore, it has been demonstrated that the color attributes, or more specifically the shifts in color attributes, can be reliably and accurately detected in an automated fashion, thus providing a quantitative instrument that retains the essential simplicity of the technique.

\section{Quantitative Schlieren Method}

The mathematical development of quantitative schlieren measurements is given in Ref. 2. The important result of this develoment is the relationship between the refractive-index distribution and the ensuing ray deflections; this result provides the basis for deflectometric methods in general and has been previously stated as Eq. (1). Implicit in this derivation is the simplifying assumption that lateral ray displacements are negligible; this is a reasonable assumption for virtually all measurements in gases, and for many liquid phase experiments as well. ${ }^{6}$ Numerous methods have been developed wherein transverse ray displacements are retained. 6,7 The resulting expressions are considerably more complex than Eq. (1) and invariably require a numerical solution. For refractive-index distributions possessing simple Cartesian or axisymmetric geometries, the inversion of Eq. (1) is relatively straightforward, as the latter case is obtained by Abel transformation. ${ }^{8}$ For asymmetric refractive-index distributions, tomographic inversions must be utilized, requiring deflec- tion data to be acquired from multiple angular fields of view. ${ }^{8}$

From the Fourier shift theorem, changes in the direction cosines of the propagating rays as they emerge from the test section appear as deflections, or lateral shifts in the plane of the filter. The magnitude of the deflections is then given by

$$
d=f_{c} \tan \theta_{\text {exit }} \approx f_{c} \theta_{\text {exit }},
$$

where $d$ is the magnitude of the deflection at the plane of the schlieren filter and $f_{c}$ is the focal length of the decollimating lens. The approximation in Eq. (2) is accurate to roughly 1 part in $10^{5}$ for most optical systems typically encountered. This relation illustrates the operation of all schlieren systems. It is immediately apparent from the above relation that the resulting measurement sensitivity is directly proportional to the focal length of the decollimator. This focal length can be varied by an order of magnitude or more, providing a large dynamic range for schlieren systems. This contrasts with interferometric methods, wherein the observed phase shifts are inversely proportional to the illumination wavelength and can thus be varied only over a comparatively narrow range.

\section{Color-Based Filtering}

A variety of filter schemes have been utilized in the color schlieren method and are summarized in Ref. 3 . The earliest report of colored schlieren images was by Toepler (ca. 1859), wherein he observed gradations in colors attributed to chromatic aberration in a field lens, effectively dispersing the color spectrum of the source image. The amateur microscopist Julius Rheinberg (1896) was the first to report the actual implementation of color-based filters. ${ }^{9}$ He described the benefit in contrast of color variations over grayscale methods and indicated the ability of color-based methods to provide both the magnitude and direction of the resulting ray deflections. The largest overall contribution to the subject was provided by Hubert Schardin (1942). His treatise on schlieren methods ${ }^{1}$ remains the most thorough reference on the subject and provides a detailed description of the color-based techniques that form the basis for the majority of techniques used today, including the. prism, lattice, and color-background methods.

The principal drawback associated with the above methods arises from the occurrence of discontinuities in the construction of the filter. Fourier transform theory indicates that discontinuous truncation of any spectrum gives rise to ringing in the reconstructed waveform. In the prism and lattice methods, these discontinuities are associated, respectively, with the edges of the aperture stop and with the opaque intercolor strips defining the lattice. In the colorbackground methods, discontinuities occur at the boundaries separating adjacent colors. These discontinuities ultimately degrade the spatial resolution of the final image, either through diffraction, truncation of the Fourier spectrum, or a combination of both. 
The lattice and color-background methods also suffer from a decrease in detection sensitivity because of the finite number of color bands or lattice strips that are employed.

As a result of these difficulties, the use of a filter with a continuous color spectrum is preferred. This use simultaneously alleviates the undesirable optical properties previously described while increasing the detection sensitivity by means of a continuous color palette. Two methods for constructing a filter of this type have been demonstrated: the wedge interference filter ${ }^{10}$ and the rainbow filter. ${ }^{4}$ Both schemes provide a continuous transfer function that associates color transmissivity with the spatial location on the filter plane. A broadband source image is thus rendered a particular hue depending on the coordinates of the filter through which it passes. Although both, in principle, are suitable for this application, the former is somewhat inconvenient in practice. For a schlieren head of fixed focal length, variations in dynamic range associated with various applications require the use of a number of filters that range in size. This is not well accommodated by wedge interference filters, whose cost of fabrication is significant. This is particularly true if more complicated geometries, such as circular filters, are desired. As we show below, achieving optimal performance in quantitative applications requires the filter transfer function to be warped to compensate for the behavior of other system components. This is extremely impractical to accommodate with a wedge filter.

An alternative to the wedge interference filter is the rainbow filter, first introduced by Howes. ${ }^{4}$ As originally implemented, the rainbow filter was fabricated by the projection of the spectrally dispersed output of a broadband xenon arc lamp onto a strip of photographic color slide film (Kodak Daylight Ektachrome, ASA 64). Radially symmetric filters were obtained by rotation of the film during exposure. The transparencies produced in this manner were far too large ( $\approx 75 \mathrm{~mm}$ in diameter) to be used as actual filters and were subsequently reproduced in a variety of sizes when the backlit original was photographed at varying distances. A double exposure was normally used during the second step of this process. After the suitably scaled image of the color filter was first recorded, a mask was then inserted to obscure all but the central portion of the filter. The second image was then overexposed, resulting in the occurrence of a clear region in the center of the final filter. When the diameter of this clear region was matched to the diameter of the image of the schlieren source, a nonperturbed condition in the test section resulted in an image free from color attributes (i.e., white). Although this proved advantageous from the standpoint of qualitative interpretation, the discontinuous boundary between the central clear region and the remaining portion of the filter led to diffraction problems identical to those previously described. Attempts to alleviate this problem through the use of a mask with soft edges were discussed in Ref. 4 but were never actually implemented.

In our development of a quantitative method, we sought to produce a filter with a color transmission function at any spatial location described by a single parameter. Additionally, this color parameter should be insensitive to absolute optical intensities. This eliminates numerous complications such as intensity fluctuations of the source, pixel-to-pixel variations in gain within a given detector array or from one detector to another, absorption or scattering of light within the test section, or section-order intensity variations resulting from asymmetric refractiveindex distributions. ${ }^{11}$ An example of a color basis that does not possess these properties is the RGB (red, green, and blue) representation provided by most conventional color video systems. The tristimulus values must be combined according to some predetermined formalism to yield the net perceived color, and each of the three values of $R, G$, and $B$ are themselves dependent on the absolute intensity. A color basis that avoids this difficulty, and the one that was selected for this application, is the relatively well-known hue-saturation-intensity (HSI) representation. ${ }^{12}$ HSI parameters are obtained from direct transformation of the RGB tristimulus values according to the following transformation equations:

$$
\begin{aligned}
I & =\frac{R+G+B}{3}, \\
S & =1-\frac{\min (R, G, B)}{I}, \\
H & =\cos ^{-1}\left\{\frac{1 / 2[(R-G)+(R-B)]}{\left[(R-G)^{2}+(R-B)(G-B)\right]^{1 / 2}}\right\} .
\end{aligned}
$$

A schematic representation of the relationship between the RGB and HSI color-space models is shown in Fig. 2; the vertex of the HSI cone is anchored at the intersection of the $R, G$, and $B$ axes, and the projections of the central axis of the cone onto the $R, G$, and $B$ axes are identical. An inspection of Eq. (5) confirms that the hue is independent of the absolute

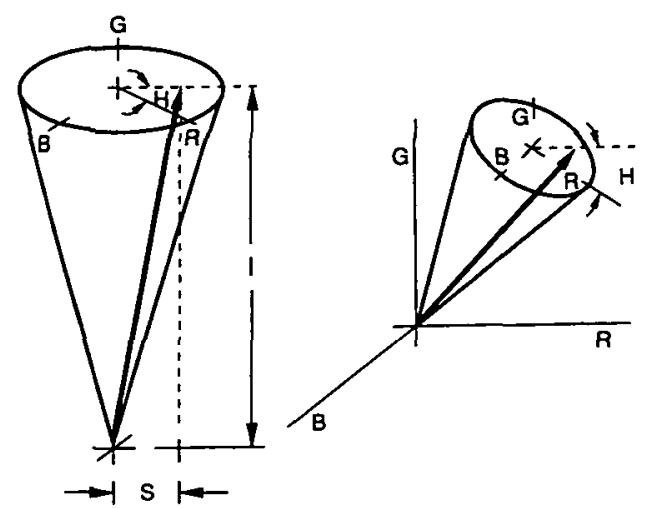

Fig. 2. Graphic representation of the HSI color space and its relationship to the $R, G, B$ tristimulus vectors. 
intensity. This can also be seen from Fig. 2; the hue, which is given by the polar angle relative to pure red, is unaffected by the total length (intensity) of the color-space vector. Thus the RGB output of a conventional color imaging array can be readily transformed in such a fashion as to produce a suitable oneparameter measure of color.

To describe a HSI filter, one must map the polar angle onto a one-dimensional coordinate. When this is done on one of the lateral dimensions of the filter plane, a Cartesian or bandlike filter is produced. Similar to a conventional knife-edge stop, such a filter is sensitive to only the horizontal (or vertical) component of deflection. When mapped onto the radial coordinate, an axisymmetric, or circular filter is produced. A filter of this type responds to the absolute magnitude of the resulting deflections but not to their orientation.

\section{Filter Fabrication and Optimization}

Although $\mathrm{H}(\mathrm{R}, \mathrm{G}, \mathrm{B})$ is single valued and independent of $I$, it is a nonlinear function of its arguments. A parametric relationship between the $\mathrm{R}, \mathrm{G}$, and $\mathrm{B}$ tristimulus values, such as the one in Ref. 13, is therefore required to achieve a distribution that is a linear function of position. This form must be empirically derived, because the inverse transformation associated with Eq. (5) does not yield a unique solution and because a HSI color-space vector corresponding to a given hue may take on any arbitrary value of saturation or intensity. The saturation is defined as the departure of the color-space vector from the perimeter of the HSI cone. Complete saturation is described by $\mathrm{S}=1$. The opposite limiting case is given by $\mathrm{S}=0$ and corresponds to equal magnitudes for each of the tristimulus values; in this case the color-space vector is coincident with the axis of the HSI cone. Although the value of $\mathrm{S}$ in this context is arbitrary, computations are simplified by choosing $S=1$. By an inspection of both Eq. (4) and Fig. 2, it can be seen that complete saturation is achieved only when one of the tristimulus values is equal to zero.

Figure 3(a) depicts the essential features of a Cartesian filter. The active or colored portion of the filter possesses the transmission function described by Eq. (6). Bounding the active portion of the filter are a black (opaque) band followed by a white (transparent) band of equal width. These bands serve as spatial indexing marks for determining the absolute position of each pixel used to produce the filter. The width of the indexing bands is chosen to match that of the source image as it appears in the plane of the filter.

Because the filter is ultimately rendered onto photographic film, the parametric relationship that provides a constant linear variation of hue becomes distorted in the development process. In addition, each color imaging array uses its own set of color separation filters or prisms, thus serving to distort further the color properties measured by that array.
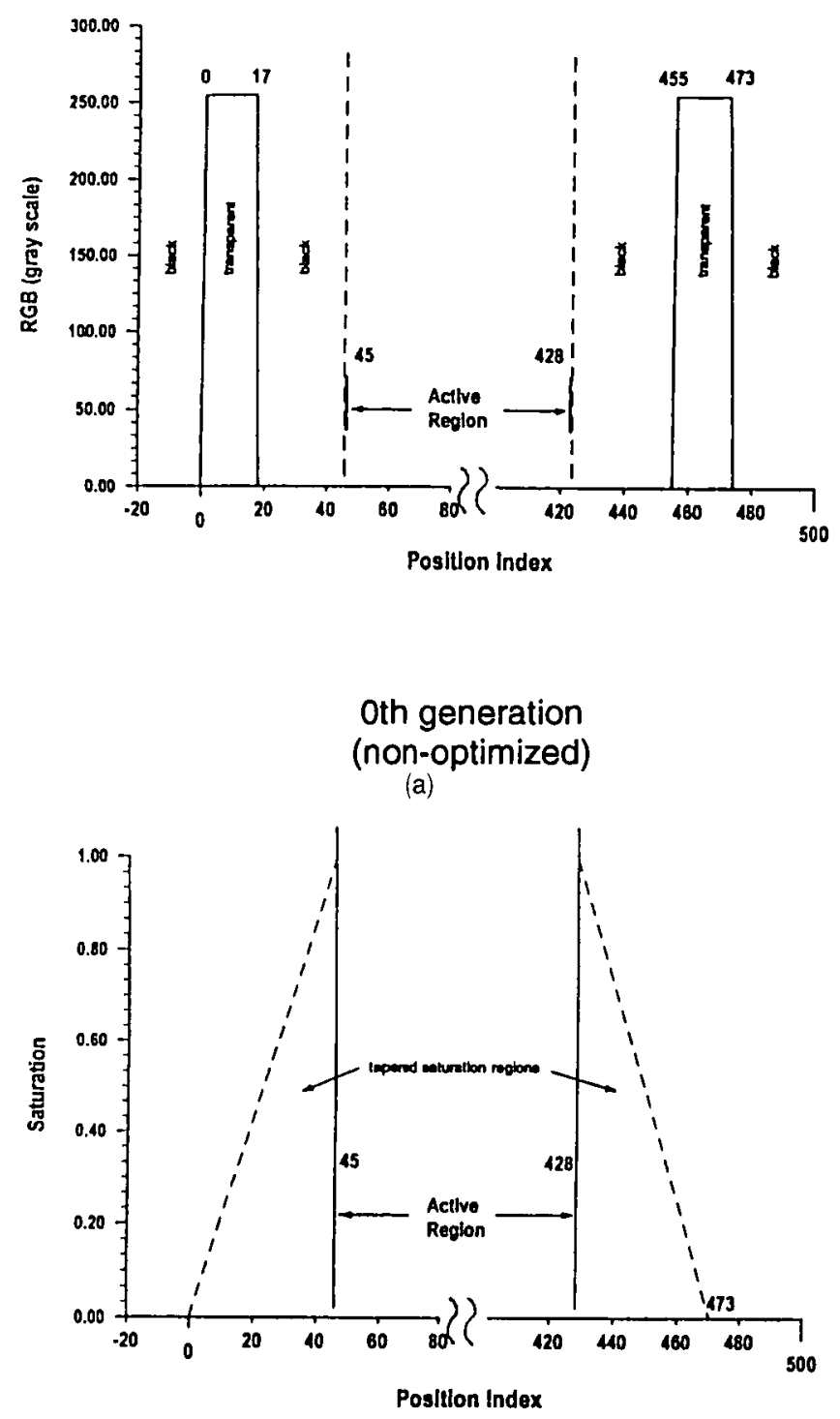

\section{1st generation \\ (optimized)}

(b)

Fig. 3. Pixel layout of a Cartesian filter: (a) the nonoptimized filter is seen with the accompanying index stripes necessary for registration; (b) the optimized filter, in which these stripes are discarded and replaced with tapered saturation zones.

This is demonstrated in Fig. 4. Figure 4(a) illustrates the spatial transmissivity function of a Cartesian filter. For the present purposes this filter is referred to as the ideal or zeroth generation. The $R$, $G$, and $B$ intensity versus position attributes used to generate this filter are specified in Ref. 13 and are shown graphically in Fig. 5. Figure 4(b) in comparison shows the effect of the considerations just described. The data in this case were obtained when the filter was laterally traversed across the Fourier plane of an actual schlieren system. It is desirable to remove this distortion; specifically, the final filter 


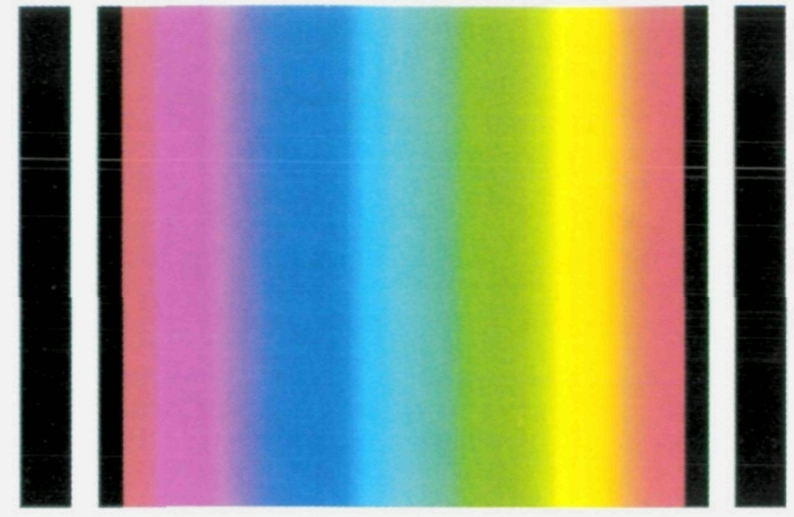

(a)
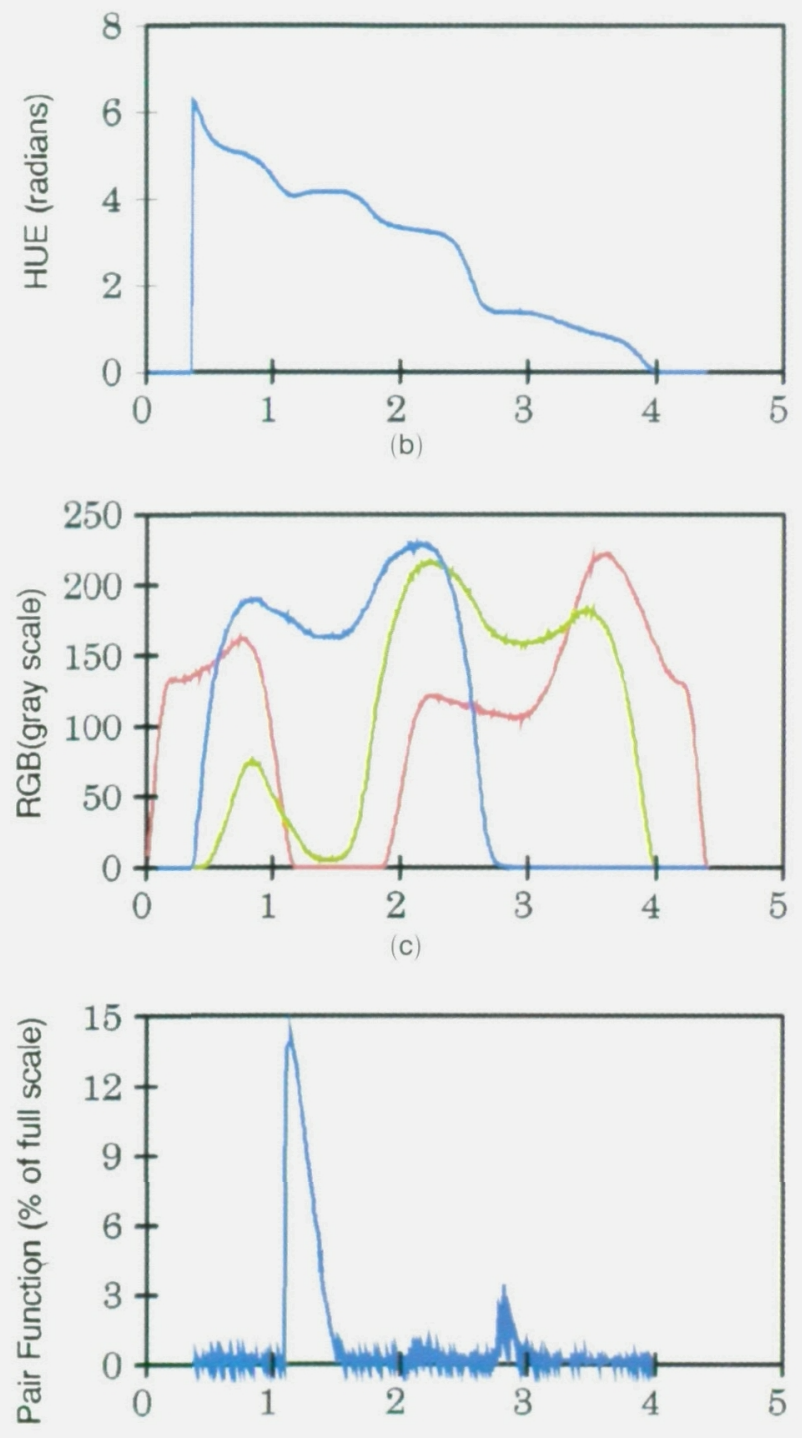

Position (mm)

(d)

Fig. 4. Original (ideal) filter. (a) Spatially dependent transmittivity function of an ideal Cartesian. (b) The hue versus position data obtained when this filter was scanned. (c) The R, G, and B values corresponding to this scan. (d) The pair function derived from these data.

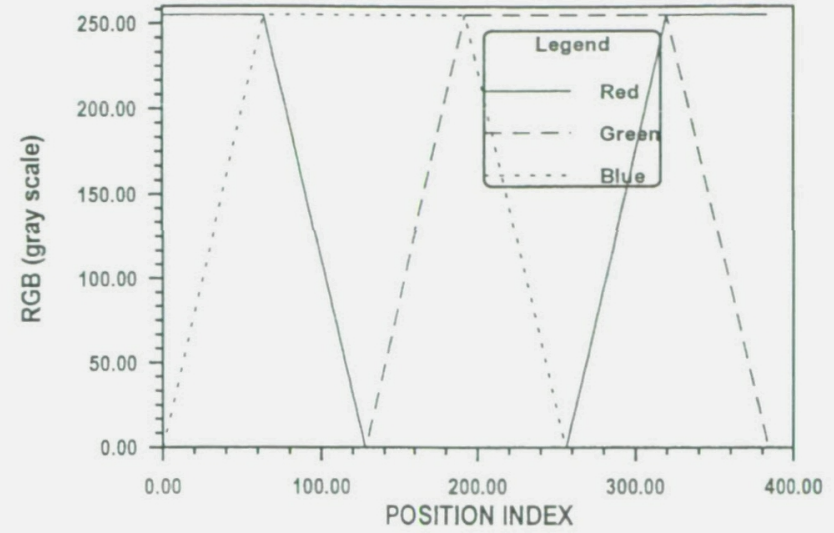

Fig. 5. R, G, and B tristimulus values used to generate an ideal filter

should possess the property that

$$
\partial \mathrm{H} / \partial x=\text { const }=-\frac{2 \pi}{X_{\max }},
$$

where $x$ is the transverse coordinate in the filter plane over which $\mathrm{H}$ varies and $X_{\max }$ is the overall dimension of the filter. As demonstrated below, departures from this behavior result in a detection sensitivity that is nonuniform over the measurement range. Although this effort has focused on filters adhering to the linear behavior described by Eq. (6), computerbased filter generation affords the flexibility to produce filters described by other relationships. One such category is logarithmic scaling, providing an increase in dynamic range while retaining sensitivity in the neighborhood of a particular operating point.

For a filter to be optimized, determining the relationship between the originally specified RGB values at each spatial location and the subsequent RGB values that are measured at that same location by the complete instrument is required. This relationship then includes the composite effects attributable to the device used to display the original filter, the photographic recording material, any color shifts incurred in the photographic development process, the color spectrum of the schlieren illumination source, and the transfer function of the detector array. Hence this relationship must be determined in situ, because Eq. (6) is not a property of the filter taken by itself but rather is a property of the filter acting in concert with the rest of the system. As stated previously, one can determine this input-output relationship by measuring the resulting value of hue while incrementally traversing the filter across the Fourier plane. One finds the absolute location of each index band by calculating the centroid of the intensity distribution that is observed as the bands traverse the source image. Under these conditions the position of these bands, and therefore the position of all the other pixels relative to these bands, can be determined with a positional error of less than one pixel.

The optimized or first-generation filter is then produced in the following manner. From Eq. (6) it 
follows that the desired value of hue at any position $\mathbf{x}$ on the final filter is given by

$$
\mathrm{H}(\mathbf{x})=2 \pi\left\{1-\frac{1}{X_{\max }}|\mathbf{x}|\right\} .
$$

The correct input value of hue is now selected from the set of measured output values obtained from the transverse scan of the zeroth-generation filter. Because the location corresponding to the occurrence of this measured value is known in absolute coordinates, the original magnitudes of $\mathrm{R}, \mathrm{G}$, and $\mathrm{B}$ used to produce this value can now be retrieved and assigned to this spatial location on the optimized filter. This procedure is then repeated for each value of hue until the entire range from 0 to $2 \pi$ has been completed. Because the RGB components at any position on the filter now correspond to actual measured values of hue, adherence to the behavior specified by Eq. (6) is now guaranteed. This is illustrated in Fig. 6. Small imperfections observed in the linearity of the hue versus position curve are attributed to variations occurring in the photographic development process, or in roll-to-roll variations of the photographic emulsion itself. This is not particularly surprising, because the zeroth- and first-generation filters are generated from different rolls of transparency film and are typically subject to photographic processing on different days. Furthermore, the occurrence of these imperfections places a boundary on the degree of linearity that can be achieved with this method. Attempts to improve the linearity by sequentially reoptimizing a first-generation filter have yielded relatively minor improvement.

In the process of remapping the ideal filter to optimize the behavior of its transmission function, two different cases are encountered. Over any given interval, the magnitude of the slope of the measured hue versus position curve may be greater or less than the desired value of $-2 \pi / X_{\max }$. The desired change in hue from one pixel to the next is given by $2 \pi / N_{p}$, where $N_{p}$ is the total number of pixels used to display the image of the filter. For those regions where the slope is shallower than the desired value, there is effectively a surplus of input data points; each consecutive triplet of input values is sequentially rejected until the output value of hue increases by $2 \pi / N_{p}$. For those regions where the slope is steeper than desired, there is an insufficient density of input data points. In this case the input values are determined by linear interpolation of the RGB values from adjacent pixels. Errors induced from this procedure are small in comparison with variations that are encountered in the subsequent photographic processing of the final filter.

Initially, both zeroth- and first-generation filters were produced when the image was displayed on a high-quality video monitor and photographed directly with a $35-\mathrm{mm}$ SLR camera. The original images were generated by a Matrox Model MVP/AT imageprocessing board. Filters of varying sizes were then obtained when the display monitor was photographed from varying distances or when camera lenses of differing focal lengths were used. This process was eventually supplanted with the use of a Matrix Instruments QCR D4/2 film recorder. This device contains an internal, high-resolution $(4096 \times 2732$ elements) flat-screen CRT and an automated $35-\mathrm{mm}$ film transport with relay optics. Although not strictly required, the use of a device of this type affords several advantages. Because it is pixel addressable, operations such as dimensional scaling can be easily implemented in software. Thus filters of varying sizes can be generated more readily and with greater accuracy. The video image processor previously described is limited to $512 \times 512$ pixel images and therefore suffers from an undesirable loss in overall pixel count when the dimensions of the displayed image of the filter are reduced. The principal advantage concerns the regions surrounding the active portion of the filter. As shown in Fig. 3(b), the index bands that are necessary for the optimization process are discarded for the final filter. Instead the active portion is suspended in the center of a large, transparent background field. Because the filter occupies the Fourier plane of the schlieren head, it should not present a limiting aperture that would truncate the spatial frequency spectrum normally passed by the remainder of the optical system. ${ }^{4}$ For most practical systems this criterion is met if the transparent background fills the internal area of a conventional $35-\mathrm{mm}$ slide mount, which is $23 \mathrm{~mm}$ across the shortest dimension. The relatively large pixel count afforded by the D4/2 makes it possible to achieve a suitably scaled filter-background combination while retaining a generous allocation of pixels for the active region.

In the initial method the transparent background is produced when the image of the filter displayed on the video monitor is surrounded with an opaque white surface roughly $1 \mathrm{~m}$ on a side. An in-thecamera double exposure is then taken; the first exposure records the colored image from the monitor. For the second exposure the white card is illuminated with either ambient room light or a photographic flash unit. Although acceptable results were obtained through the use of this technique, the generation of the transparent background field by software is far more convenient. Although a film recorder of the type reported here is relatively costly, it is not necessary to purchase such a device to accomplish the procedures of digital fabrication, optimization, and scaling. Numerous commercial services are available for providing access to high-quality film recorders at extremely minimal cost. The data composing the filter image can be relayed by means of electronic file transfer, with the resulting transparency printed and delivered within $24 \mathrm{~h}$. Because filters produced in this manner are quite inexpensive, it is practical to fabricate a selection of filters of varying dimensions to accommodate numerous experiments that possess a large dynamic range in angular deflections.

Because sharp discontinuities in the Fourier plane give rise to ringing in the final image, the provision of 
a smooth transition region between the colored portion of the filter and the transparent surround is desirable, as shown in Fig. 6(a). The transition region couples the end points of the filter to the background by exploiting the $S$ (saturation) variable. Because all colors lie on the surface on the HSI cone, $S$ is by definition equal to 1 . The background corresponds to the condition of $S=0$ and is coupled to the end points of the filter when $\mathrm{H}$ is held constant while $S$ slowly decreases from one to zero. At the end points of the active region $\mathrm{H}$ takes on the values of zero and $2 \pi$, corresponding to the color of pure red. It should be noted that on an optimized filter the hues at the extrema of the filter may depart slightly from pure red. Thus, by bleeding in appropriate amounts of red, green, and blue, the value of $\mathrm{H}$ remains fixed while providing a continuous transition to the surrounding field. Light passing through the transition region can easily be distinguished from actual data when the value of $S$ is checked for all the pixels wherein $\mathrm{H}$ is equal to either 0 or $2 \pi$.

\section{Source and Optics}

An essential element in the implementation of this technique is a source that exhibits suitable spatial and spectral stability. Variations in the former have been observed in those applications in which the output of an arc lamp is imaged directly onto an entrance aperture or pinhole. Because the spatial location of the cathode can drift over a time scale of 10-20 min, large intensity variations are observed in the plane of the detector as the image of the source and the entrance pinhole effectively become mismatched. This proved to be an insurmountable problem, for example, with the Sylvania C-10-P zirconium arc lamp. This difficulty could not be alleviated by the removal of the entrance pinhole, because drift in the spatial location of the source is also manifest as a drift in the source image in the filter plane, which gives rise to fluctuations in the hue of the final images, thus corrupting the entire system.

Spectral stability is, of course, key. The spectral content in this case refers to the value of hue that is computed from the measured values of $\mathrm{R}, \mathrm{G}$, and $\mathrm{B}$ according to Eq. (5). As discussed, transformation Eqs. (3)-(5) decouple the values of hue and intensity. Thus, in theory, intensity stability of the source is not a requirement. Variations of more than a few percent, however, are problematic because of the quantization error associated with the frame digitizer. If one tristimulus component comprising a given hue is very small, an intensity fluctuation of more than one least-significant bit will effectively cause that component to vanish and therefore alter the computed value of $\mathrm{H}$. The ideal situation therefore corresponds to a source whose absolute intensity fluctuations result in a variation of less than one half of one leastsignificant bit as presented to the analog-to-digital converter of the frame digitizer. This condition has been realized with an actively stabilized xenon arc lamp (ILC Model 131). Although we have found that

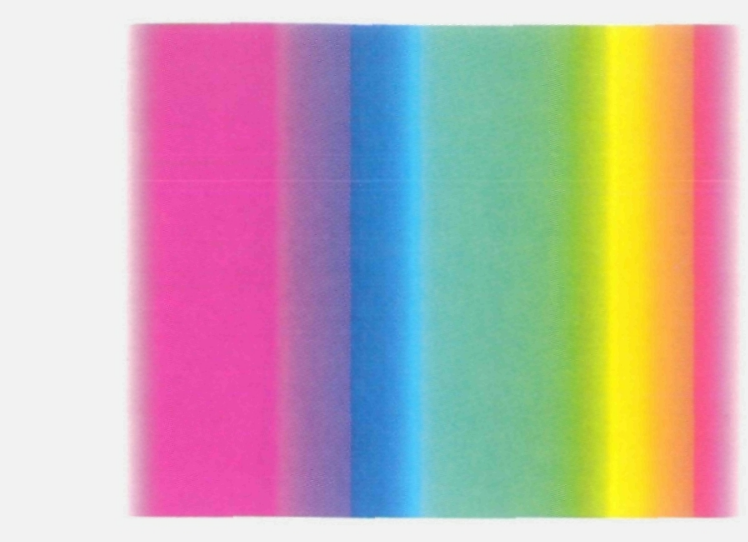

(a)
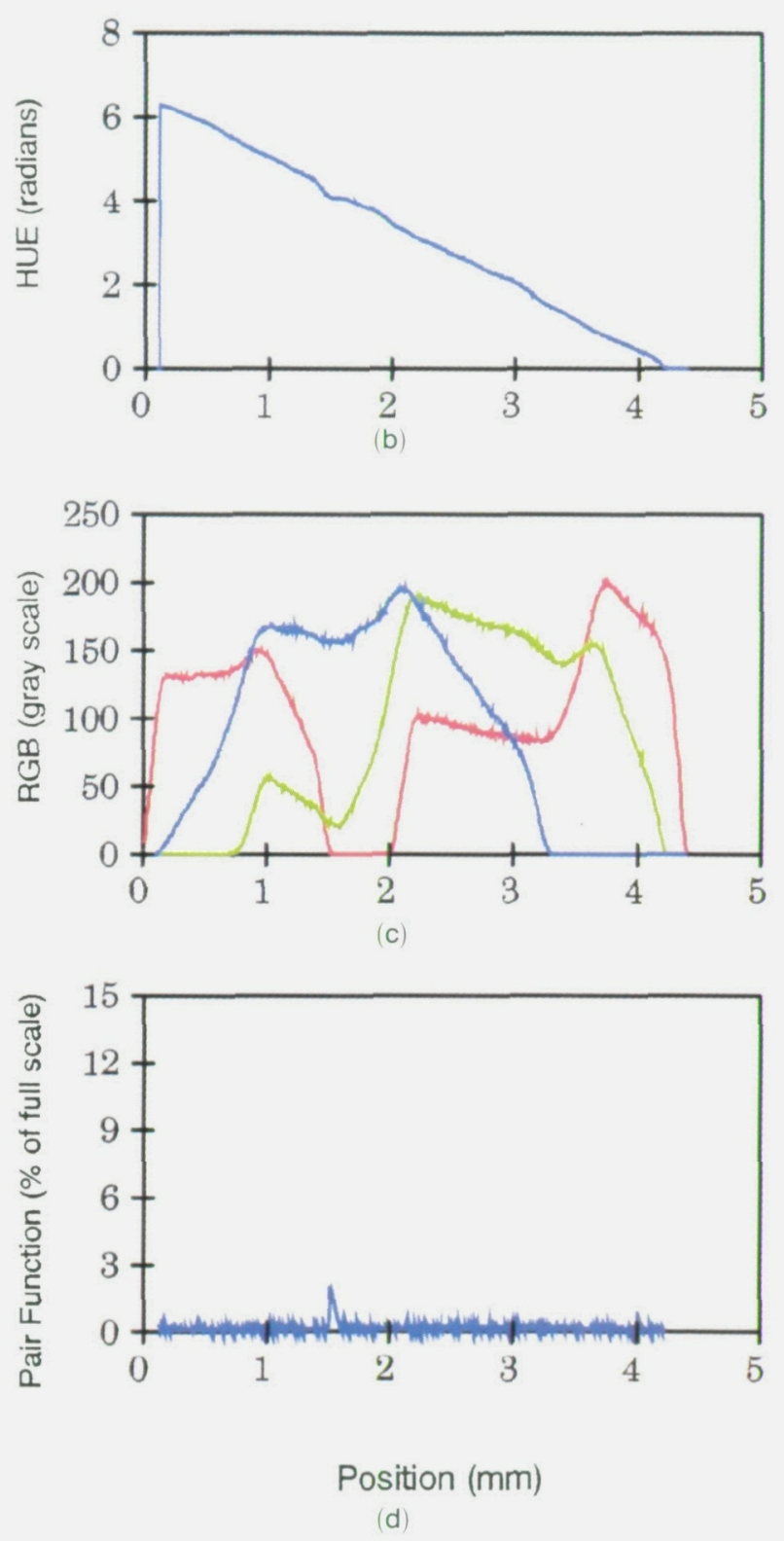

Fig. 6. Optimized filter. (a) Spatially dependent transmittivity function of an optimized filter, showing the tapered saturation zones. (b) The resulting hue versus position data obtained when this filter was scanned. (c) The associated tristimulus components. (d) The pair function derived from these data. 
this source meets our requirements, similar devices are available from other vendors. Stabilization is achieved by the collection of a fraction of the luminous output with a photodiode detector, the output of which is suitably amplified and used as a modulation signal to control the arc current in a dc-controlled power supply. Without the presence of feedback control, the luminous output of the arc lamp exhibits broadband noise, spikes, and long-term drift. The inclusion of feedback stabilization has been demonstrated to reduce all such behavior to a relative variation of less than $0.1 \%{ }^{14}$ Stabilization of the output intensity has the fortuitous consequence of stabilizing the output hue as well, presumably through the regulation of the color temperature of the arc. Measurements of the output hue demonstrate a standard deviation of typically $2 \times 10^{-3}$, relating to both short-term fluctuations and long-term drift. This represents a factor of $2-4$ improvement in terms of noise performance relative to the unstabilized configuration. The reduction in long-term drift is even more considerable but varies from one lamp to another, and with the age of an individual lamp as well. The fact that the measured variance in hue is considerably less than that associated with the absolute intensity is directly attributable to the decoupling provided by the transformation equations. This is shown graphically in Fig. 7, wherein the tristimulus values tend to exhibit synchronous variations, thus imparting only slight variations to the computed value of $\mathrm{H}$.

In addition to its favorable performance characteristics, the ILC Model 131 xenon arc is configured to launch its output directly into a multimode optical fiber. This is accomplished by an internal sapphire rod that functions as a collection light pipe. ${ }^{15}$ The exit face of the sapphire rod is polished to form a positive lens and provides a convergent exit cone of approximately $f / 2$ with a minimum diameter of 200 $\mu \mathrm{m}$ FWHM. This is well suited to couple directly into a fiber with a diameter of $200 \mu \mathrm{m}$ or greater, thus providing flexibility in the location of the source. In general, a $200-\mu \mathrm{m}$-diameter fiber followed directly by a pinhole or variable slit that defines the entrance aperture of the optical system has been used. The

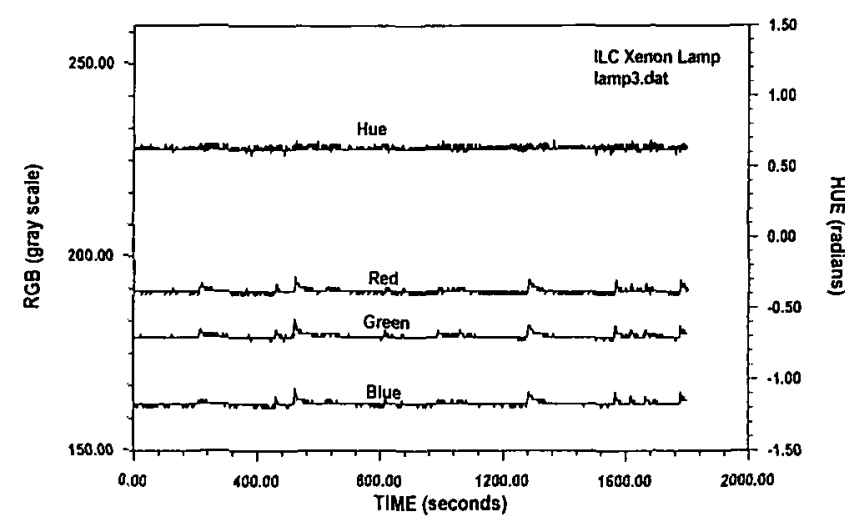

Fig. 7. Temporal variations in the $\mathrm{R}, \mathrm{G}$, and $\mathrm{B}$ components and hue of a stablized xenon arc lamp. variable slit is used for those applications employing a bandlike filter; a pinhole is used in conjunction with radially symmetric filters. The source has more than sufficient brightness to saturate a conventional color imaging array, so that masking the exit face of the fiber to define the proper entrance aperture still provides more than adequate source strength.

Schlieren optical systems have been described in detail elsewhere., ${ }^{1,5,16}$ All require a perfect image of the source at the plane of the filter. More specifically, the filter plane must represent the true Fourier transform of the test section midplane. If this condition is violated, Eq. (2) is not valid, and hence quantification of Eq. (1) is precluded. This technique places further demands on the optical system, because broadband illumination is utilized. Thus, in addition to geometric aberrations, chromatic aberrations must be considered as well. Although reflective systems eliminate the latter concern, typical elements may present other difficulties. For example, off-axis paraboloidal (OAP) mirrors form an astigmatic image of the source on axis but produce off-axis coma in the final image. The net result is that the effective focal length of the schlieren head varies over the object plane. For the case of a 75-mm-diameter, $f / 6$ OAP mirror with an off-axis distance equal to its radius, for example, this effect produces a maximum variation of the order of $2 \%$. This variation can be accommodated by a priori calibration of the system by the use of a wellcharacterized standard. OAP mirrors have the added disadvantage, however, of being somewhat costly. The expense associated with increasing the field of view of the test section beyond the range of 75-100 $\mathrm{mm}$ can become excessive. Using on-axis paraboloidal mirrors in an off-axis configuration is somewhat more cost effective but produces an astigmatic image of the source, making it unusable for applications wherein a radially symmetric filter is desired. Cylindrical lenses can be used to correct this astigmatism but in turn introduce unwanted chromatic aberrations. The results contained in this paper were obtained with a system based on corrected spherical primary mirrors. ${ }^{17}$ The present configuration provides a $100-\mathrm{mm}$ field of view and is quite compact. The optical prescription is fairly complex, containing 28 surfaces in each of the transmitting and receiving sections. Although it is constructed from commercially available off-the-shelf components, a nontrivial degree of expertise in optical system design is required to assemble and align the individual elements properly.

\section{System Performance}

To provide a figure of merit that describes the performance of the complete system, a function was constructed known as the pair function and is denoted by $P(\mathbf{x}, \sigma)$. If an unperturbed ray bundle traverses the test section and intersects the filter at an initial coordinate $\mathbf{x}$, then the value of the pair function at that point describes the minimum dis- 
tance in the filter plane by which the ray bundle must be deviated to measure an unambiguous change in the value of $\mathrm{H}$. A change in the value of $\mathrm{H}$ is deemed to be unambiguous when it exceeds the quantity $\sigma_{T}$, the standard deviation of the fluctuations in hue as measured for the complete system. In addition to fluctuations directly attributable to the source, $\sigma_{T}$ also includes the effect of fluctuations in the spatial transmittivity function of the filter. The latter was determined from measurements of the distribution of $\mathrm{H}$ that are observed when a Cartesian filter is translated in a direction normal to the active coordinate, i.e., along a locus of constant color. This measured distribution then also contains any effects caused by the illumination source as well. Measured values of $\sigma_{T}$ are typically of the order of $1.6 \times 10^{-2}$, representing a deviation relative to full scale of $0.25 \%$. This value of $\sigma_{T}$ is essentially 1 order of magnitude larger than $\sigma$ for the source taken by itself.

Figure 4(d) depicts $P(x, \sigma)$ corresponding to a typical zeroth-generation filter and illustrates the rationale for the filter optimization process. In particular, the hue versus position curve corresponding to the zeroth-generation filter contains several regions where the slope flattens. This indicates that over these particular portions of the filter, the measured value of hue exhibits relatively weak variation with position. The local sensitivity is proportional to $\partial \mathrm{H} / \partial x$ and is reflected in the value of the pair function at this location. A comparison of Figs. 4 and 6 demonstrates this point; the value of $P(\mathbf{x}, \sigma)$ at a particular spatial location is inversely proportional to the magnitude of the slope of the hue versus position curve at this same location. By forcing the spatial values of hue to be in compliance with that specified by Eq. (6), one can produce a pair function of reasonably constant value and hence uniform sensitivity across the entire dynamic range. Using this process, we have been able to produce and optimize filters routinely such that $P(\mathbf{x}, \boldsymbol{\sigma})$ has a value at all locations of $0.2 \%$ full scale or less. This criterion provides a more conservative assessment of the sensitivity of the overall system than would be indicated by the rms, average value, or probability density function of the pair function.

Interestingly, the measured value of $\sigma_{T}$ is influenced by the size of the source image as it appears in the plane of the filter. When the source image becomes small, approaching the diffraction limit of the optical system, spatial noise in the filter itself dominates and degrades the resulting pair function. Increasing the size of the source image improves this situation, but at the sacrifice of overall dynamic range. This statement can be better understood in the context of Fig. 3. When ray deflections become large enough to approach the extrema of the active portion of the filter, the source image begins to spill over into the tapered regions where $\mathrm{H}$ equals 0 or $2 \pi$, and where $S$ varies parametrically from 1 to 0 . A threshold criterion for the value of $S$ (typically of the order of 0.75 ) is used to verify whether these values of
$\mathrm{H}$ correspond to the active portion of the filter where $S$ is close to one. Values of $S$ below the threshold are assumed to correspond to rays traversing the tapered transition regions and are discarded. As the size of the source image is increased, this spillover occurs earlier and begins to encroach on the usable values of $\mathrm{H}$ that lie near the extrema of the filter. In practice, a source image corresponding to $5 \%$ of the size of the overall filter represents a realistic balance between these two factors. In contrast to conventional knifeedge schlieren methods, the sensitivity and dynamic range are now essentially decoupled from the size of the source image. ${ }^{5}$

The sensitivity afforded by this method is notable when viewed in the context of earlier studies by Howes, ${ }^{18}$ wherein a comparison was made between this method and conventional Mach-Zehnder interferometry. Because the two instruments measure fundamentally different quantities, the comparison addressed a specific application, namely, the measurement of a unidirectional refractive-index distribution corresponding to a supersonic boundary layer. In light of the results described here and recent advances in the area of the evaluation of interferometric images, several numeric values cited by Howes must be modified to provide a valid comparison. Specifically, the measurement sensitivity pertaining to the rainbow schlieren is specified to be $0.2 \%$ of full scale, as described in the preceding paragraph. For this quantity Howes previously specified a value of $20 \%$ full scale. The latter value was derived from published data relating to the colorimetric sensitivity of the human eye. It is interesting to note the distinction that is made between the threshold and measurement sensitivities of the eye. The latter is utilized in this regard but differs by the former by a factor of 40:1. The results described in this paper thus imply a measurement sensitivity that is roughly twice that of the threshold sensitivity of the human eye. For the measurement sensitivity of the interferometer, Howes cited the value of $n / 10$. More recent research in the area of quasi-heterodyne interferometry has provided a value of $N / 100 . .^{19,20}$ Inserting these values into the derivation provided by Howes indicates that the measurement sensitivity for the rainbow schlieren exceeds that of the Mach-Zehnder interferometer by a factor of 13.3:1. The conclusion drawn from this comparison is that for this specific measurement, the schlieren apparatus is capable of resolving a minimum difference in refractive index that is 13.3 times less than that that can be resolved by the interferometer.

The process of scanning, optimizing, and evaluating the performance of a given filter is best accomplished with a fixed-system configuration. As previously indicated, the fundamental explanation for the success of the optimization strategy is that it precisely accounts for each element of the process. The forced adherence to Eq. (6) factors in the characteristics of the illumination source, the transmission properties of the filter material, the spectral sensitiv- 
ity of the imaging array, and so forth. If one or more elements are changed in the interim, this behavior is no longer guaranteed. This statement is not, however, as restrictive as it might first appear. Frequently, the substitution of specific elements has a relatively minor impact on the behavior of $P(\mathbf{x}, \sigma)$, for example, the substitution of imaging arrays. When a first-generation filter is scanned by the use of an array that is different from the one used to produce and optimize the same filter, the resulting hue versus position curve is not significantly altered. In any event, it is clear that optimal performance is achieved when all the elements of the system are retained. Because various components, most notably the illumination source, do exhibit long-term drift, updating the hue versus position lookup table at periodic intervals is advisable. Without the production of a new filter, the net result of this process is the maintenance of accuracy at the expense of uniform sensitivity.

The majority of the results described herein that concern evaluating, optimizing, or regenerating the lookup table for a given filter were obtained by the traversal of the filter relative to the source image. Even through the use of programmable micropositioners, this is a time-consuming process. Accomplishing all these objectives in a single-shot fashion is possible through the use of a suitably constructed calibration standard, which generally takes the form of a weak spherical lens. When applied to Cartesian filters, an appropriate choice of radius of curvature for the standard results in a test section image that essentially replicates the appearance of the filter itself. The hue versus position lookup table, for example, can be generated or updated in a single-shot fashion when one row of pixels is read out from the imaging array. The disadvantage of this procedure is that the lookup table can contain only as many independent measurements of hue as the array has pixels in that dimension. In contrast, a programmable micropositioner can be made to move in steps of almost arbitrarily small increments. This limitation is virtually insignificant because a given filter containing of the order of 500 independent values of hue is served perfectly well by an imaging array containing as many or more pixels in a transverse dimension. The evaluation of a pair function presents a different situation, because the position or deflection increments must be smaller than the smallest length scale for a change in hue that is anticipated. Another disadvantage of this strategy is that these standards typically must be custom made, because lenses of this very low power are generally not commercially available. Ideally the radius of curvature is chosen to match the dimensions of a specific filter, the implication being that a variety of filter sizes must be accompanied by a commensurate set of calibration standards. This is not as inconvenient as it might at first seem, because it is a reasonably simple exercise for one to construct a variable focal length standard by varying the relative spacing be- tween as few as two commercially available spherical elements.

\section{Applications and Discussion}

As an illustration of the utility of this method for quantitative applications, several examples are provided. Typically the quantity of interest is the angular deflection of a specific ray bundle and is related to measurements of lateral shifts in the filter plane by means of Eq. (2). The constant of proportionality that relates these two quantities is the effective focal length of the decollimating lens, and thus this parameter must be accurately known. The best approach is to measure this focal length directly. This can be easily accomplished with any object of known refractive index and wedge angle. A convenient method for achieving this is through the use of a gas wedge. One configuration for such a wedge is shown in Fig. 8 . Two optical flats form the faces of a right-angle prism, the interior of which is filled with a suitable gaseous medium. The relationship between the gas pressure inside the wedge and the resulting ray deflections is easily calculated. A first-order regression of the measured lateral deflections in the filter plane as a function of gas pressure provides an accurate determination of the effective focal length of the decollimating lens. Typical data demonstrating this process are shown in Fig. 9. When nitrogen is used as the fill gas, the angular deviation at vacuum conditions relative to atmospheric pressure is of the order of 0.5 mrad.

Figure 10 is the two-dimensional slope surface of a 75-mm-diameter precision BK-7 optical flat measured by the use of quantitative rainbow schlieren deflectometry. The effective focal length of the decollimating lens was first determined by the use of a gas wedge of the type previously described. The average value of the resulting ray deflections was $0.580 \mathrm{~mm}$, corresponding to an angular deviation of $0.226 \mathrm{mrad}$ or a wedge angle of 46.7 arcsec. As a consequence of measuring the gradient of the refractive index, the slope at each point is determined independently and does not require the differentiation of experimental data, as would be the case if the same optical flat was measured by the use of interferometric techniques. In addition, the uniformity of the hue over the image

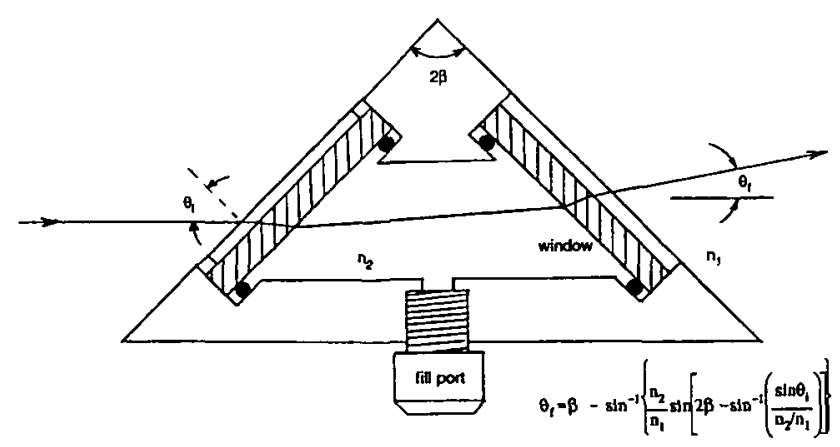

Fig. 8. Configuration of a gas-filled wedge for the generation of known ray deflections. 


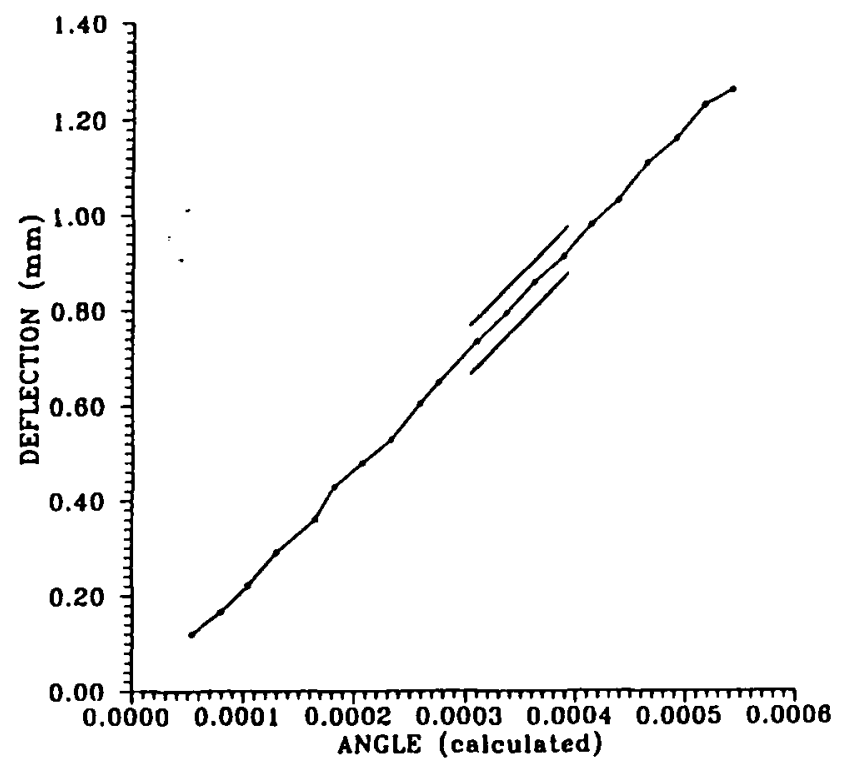

Fig. 9. Plot depicting predicted deflection angles as a function of gas pressure versus measured ray deflections at the plane of the filter for the gas wedge of Fig. 8. The best fit to the slope of this line corresponds to the focal length of the decollimating lens. The error bars represent the accuracy of the gauge used to determine the gas pressure.

of the object provides a direct indication of the surface flatness.

The second example (Fig. 11) depicts the temperature field in a radiantly heated pool of 10-cS (centistokes) silicon oil. The pool is $12.50 \mathrm{~mm}$ in depth and $44.45 \mathrm{~mm}$ in extent along the direction of the optical axis. The radiant flux is supplied by a $\mathrm{NiCr}$ heating wire of $0.203-\mathrm{mm}$ diameter, located a distance of $2.0 \mathrm{~mm}$ above the liquid surface. The wire is collinear with the optical axis and is terminated with low-impedance copper leads to minimize temperature end effects beyond the extent of the test section. Because the rainbow schlieren provides a measure of gradients in refractive index, the determination of absolute temperatures proceeds in the following manner. First the intrinsic material property describing $\partial \mathrm{n} / \partial \mathrm{T}$ is utilized to convert refractive-

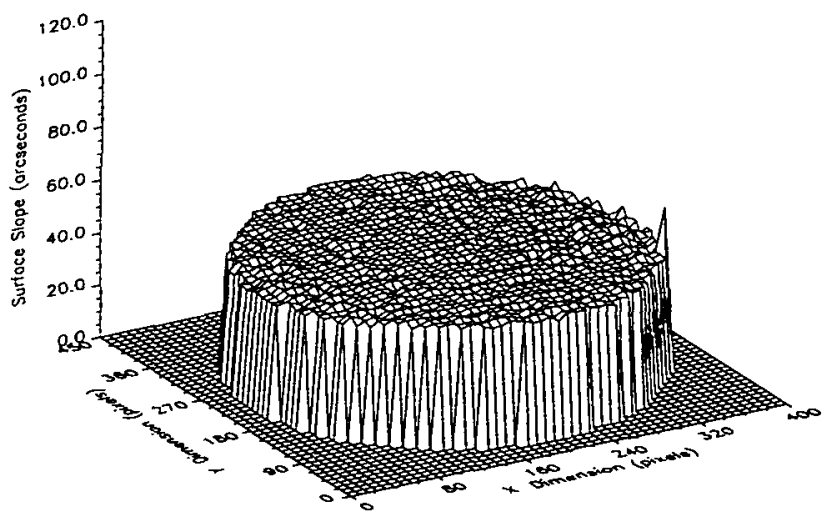

Fig. 10. Plot of the surface slope of a 75-mm-diameter BK-7 glass window. index gradients to temperature gradients. The temperature gradients are then integrated from a boundary or region of known temperature to evaluate the distribution throughout the remainder of the field. In the case shown here, this was accomplished when a reference thermocouple was embedded in the bottom surface of the pool. A Cartesian filter was used and oriented horizontally to provide a measurement of only the vertical component of the refractiveindex gradient. The gradients were then integrated in a vertical fashion from the boundary defined by the bottom surface of the pool. For this particular combination of optical path length and optical properties of the test liquid, the measurement sensitivity is of the order of 1 mdeg $C$. This figure neglects any image-plane distortions that are produced, particularly in those regions where terms of second order or higher are nonnegligible, ${ }^{11}$ as is the case in the immediate vicinity of the radiantly heated liquid surface. Regardless, the resulting sensitivity is substantial, particularly in light of the fact that the decollimating optics in this case has a modest focal length of only $304 \mathrm{~mm}$.

There are additional factors limiting the fundamental sensitivity of the quantitative rainbow schlieren. In principal, a hue space derived from a combination of tristimulus components, each of $n$-bit accuracy, should provide in total $3\left(2^{n}\right)^{2}$ independent values. For the present situation in which $n=8,1.966 \times 10^{5}$ independent values of hue should be realizable, corresponding to a sensitivity in terms of percentage of full scale of $5 \times 10^{-4}$. A sensitivity of this order is clearly not achieved and exceeds that described here by a factor of 400:1. In terms of effective bit resolution, the relation $n_{\text {eff }}=\left[\ln \left(\mathrm{H}_{\text {tot }} / 3\right)\right] / 2 \ln 2$ yields an effective resolution of 3.69 bits. $\mathrm{H}_{\text {tot }}$ is the total number of independently realizable hues and is roughly equal to 500 at present. It is clear from this discussion that the color imaging array and associated analog-todigital converter do not significantly affect the performance of the overall system. Signal-to-noise ratios in excess of $49 \mathrm{~dB}$ are routinely available from even the most modestly priced color CCD arrays and frame grabbers. Short-term color stability in the source is also not a limiting factor. The achievable sensitivity is thus traceable to the attributes of the rainbow filter itself.

Although an exhaustive study of available photographic media has not been performed, the particular transparency film selected for this application (Kodak Ektachrome $100 \mathrm{HC}$ ) was chosen for its superior properties of color saturation, sharpness of image, and fine grain. Densitometry curves for each of the corresponding tristimulus components demonstrate a linear relationship between exposure and optical density over a dynamic range in excess of $100: 1$; color saturation is therefore also not a consideration. The present value of essentially 500 corresponding to $\mathrm{H}_{\text {tot }}$ was achieved with a filter that was generated with only 383 hue values. This is due to the source image covering a number of pixels effectively present on the 


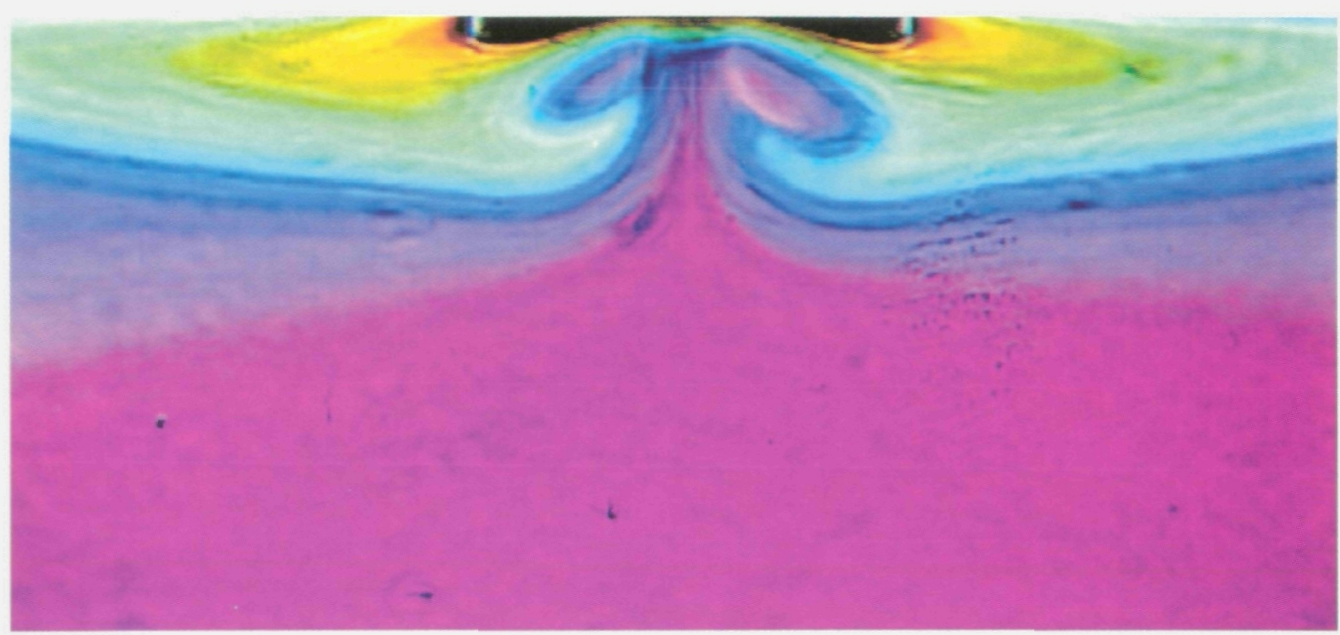

(a)

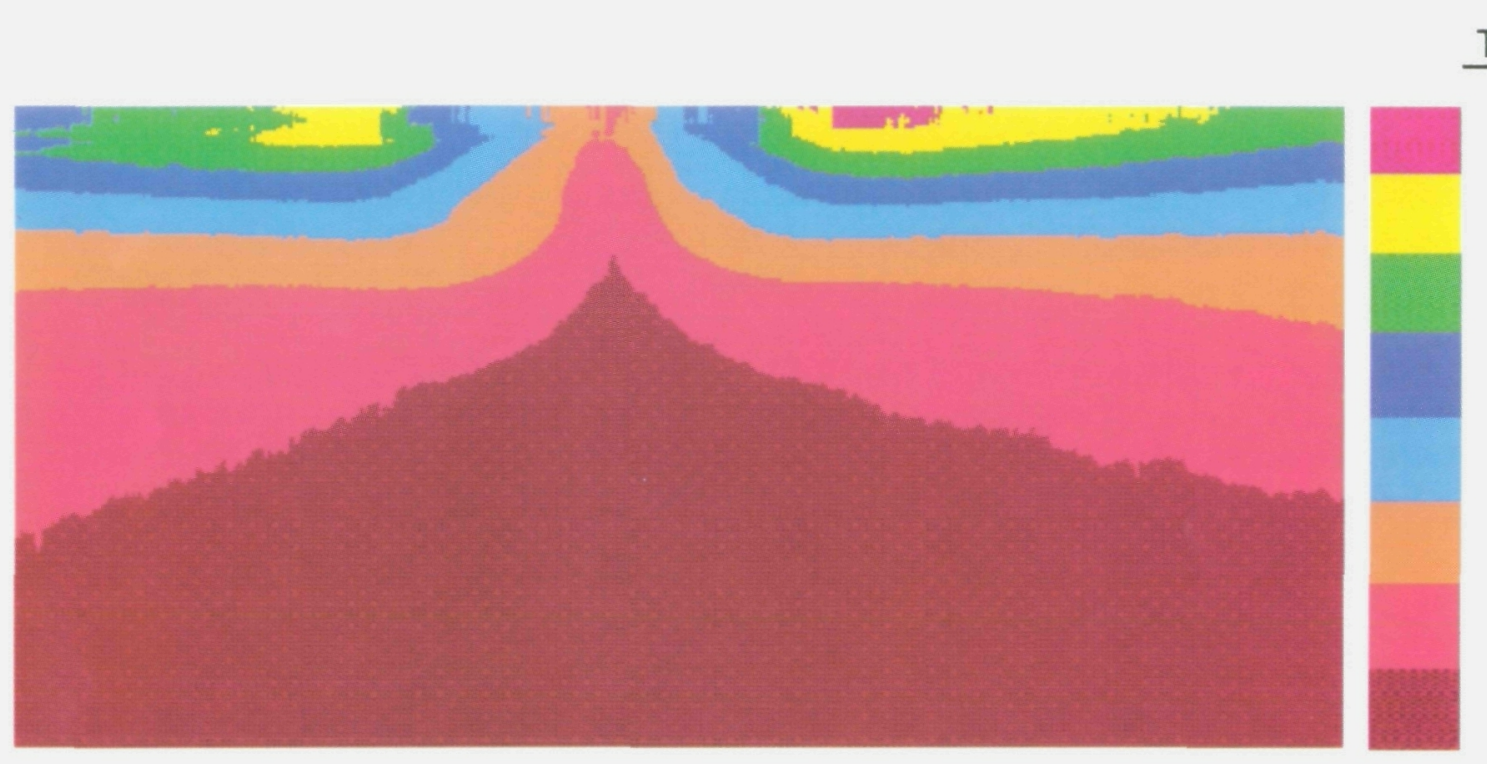

Temp. ${ }^{\circ} \mathrm{C}$

23.30

23.00

22.70

22.40

22.10

21.80

21.50

21.20

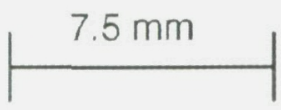

(b)

Fig. 11. (a) Raw data and (b) temperature field reconstruction of a radiantly heated pool of 10-cS silicon oil.

filter, with the resulting value of hue being computed from the integral of the transmittivity function evaluated over the spatial extent of this image. Lateral translations of the source image of subpixel magnitude correspondingly weight the contribution of any given pixel on the filter to the total value of this integral. As is demonstrated by the pair function, subpixel translations of the source image, and thus the hue shifts generated by these translations, are indeed detectable. The number 383 arose from constraints in the hardware originally used to produce the image of the filter. The D4/2 film recorder described in Section 4 affords a pixel count of approximately ten times the original amount, but this increased resolution is not overly beneficial.

Consider a filter of $4 \mathrm{~mm}$ overall dimension, which is typical for many of the applications and system focal lengths previously described. Each pixel in the original image of the filter will now occupy a region of approximately $10 \mu \mathrm{m}$ on a side as it appears in the final filter transparency. This is essentially the resolution limit of the photographic material itself. Thus a significantly improved performance will not be achieved unless filters of much larger dimensions are produced. Because the resulting angular deflections are intrinsic to the phenomena under investigation, the use of these larger filters demands a commensurate increase in the focal length of the decollimating lens, as seen in Eq. (2). There are practical limits to the focal lengths that can be utilized in most situations, even when folded or compound optical designs are considered. From this perspective an expected improvement in sensitivity of more than half an order of magnitude is somewhat impractical. Even if finer 
grained films were to become available (a situation that is somewhat unlikely given the present shift toward electronic imaging technologies), the production of the required photographic images would place excessive demands on the photographic optics. The resolution of an ideal $f / 2$ photographic lens as specified by the Rayleigh criterion is approximately 2.4 $\mu \mathrm{m}$, which exceeds the spatial resolution of typical emulsions by only a factor of 4 . Thus, although this method provides appreciable sensitivity through extremely simple and relatively inexpensive means, its fundamental sensitivity is not likely to be appreciably improved. A possible exception to this statement could arise through the use of continuously graded interference filters of the type discussed in Section 3. The relatively high cost associated with the fabrication of filters of this type, however, combined with the intractability of successively optimizing their performance renders this a relatively unattractive option.

\section{References}

1. H. Schardin, "Schlieren methods and their applications," NASA Rep. TT-F-12731 (NASA, Greenbelt, Md.; 1970) [Ergeb. Exakten Naturwiss. 20, 303 (1942)].

2. W. Merzkirch, Flow Visualization (Academic, New York, 1974), pp.71-102.

3. G. S. Settles, "Color schlieren optics-a review of techniques and applications," in Flow Visualization, W. Merzkirch, ed. (Hemisphere, New York, 1982), Vol. 2, pp. 749-759.

4. W. L. Howes, "Rainbow schlieren and its applications," Appl. Opt. 23, 2449-2460 (1984).

5. L. A. Vasil'ev, Schlieren Methods (Israel Program Scientific Trans., New York, 1971), pp. 10-11.

6. C. M. Vest, "Interferometry of strongly refracting axisymmetric phase objects," Appl. Opt. 14, 1601-1606 (1974).

7. E. E. Anderson, W. H. Stevenson, and R. Viskanta, "Estimat- ing the refractive error in optical measurements of transport phenomena," Appl. Opt. 14, 185-188 (1974).

8. C. M. Vest, Holographic Interferometry (Wiley, New York, 1979).

9. J. Rheinberg, "On an addition to the methods of microscopical research, by a new way optically producing color-contrast between an object and its background, or between definite parts of the object itself," J. R. Microsc. Soc. 16, 373-388 (1896).

10. J. R. Meyer-Arendt and H. Appelt, "Microscopic color schlieren system using a wedge interference filter," Appl. Opt. 15, 2017-2019 (1976).

11. W. L. Howes and D. R. Buchele, "Optical interferometry of inhomogeneous gases," J. Opt. Soc. Am. 56, 1517-1528 (1966).

12. R. N. Strickland, C. Kim, and W. F. McDonnell, "Luminance, hue, and saturation processing of digital color images," in Applications of Digital Image Processing IX, A. G. Tescher, ed., Proc. Soc. Photo-Opt. Instrum. Eng. 697, 286-292 (1986).

13. R. C. Gonzalez and P. Wintz, Digital Image Processing (Addison-Wesley, Reading, Mass., 1977), p. 177.

14. A. Pebler and J. M. Zomp, "Stabilizing the radiant flux of a xenon arc lamp," Appl. Opt. 20, 4059-4061 (1981).

15. R. Roberts, "Cermax small fiber illuminating lamp LSX 130," Engineering Note 183 (ILC Technology Inc., Sunnyvale, Calif., 1988).

16. D. W. Holder and R. J. North, Schlieren Methods (Her Majesty's Stationery Office, London, 1963).

17. D. R. Buchele and D. W. Griffin, "Compact color schlieren optical system," Appl. Opt. 32, 4218-4222 (1993).

18. W. L. Howes, "Rainbow schlieren vs Mach-Zehnder interferometer: a comparison," Appl. Opt. 24, 816-822 (1985).

19. B. Ovryn and E. M. Haake, "Temporal averaging of phase measurements in the presence of spurious phase drift: application to phase-stepped real-time holographic interferometry," Appl. Opt. 32, 147-154 (1993).

20. R. Dändliker and R. Thalmann, "Heterodyne and quasiheterodyne holographic interferometry," Opt. Eng. 24, 824826 (1980). 